\title{
Inhibition of miR-21 promotes cell apoptosis in oral squamous cell carcinoma by upregulating PTEN
}

\author{
YANLIANG ZHENG ${ }^{*}$, JINGFANG XIE* ${ }^{*}$ FANGFANG JIANG ${ }^{*}$, YUNYUN LI, GUANLIN CHANG and HAIQING MA \\ Department of Stomatology, Jining No. 1 People's Hospital, Jining, Shandong 272011, P.R. China
}

Received April 6, 2018; Accepted August 10, 2018

DOI: $10.3892 /$ or.2018.6663

\begin{abstract}
MicroRNA-21 (miR-21) has been identified as an oncogene and confirmed to serve an important role in carcinogenesis in various types of cancer. However, the effect and mechanism of miR-21 in oral squamous cell carcinoma (OSCC) has not been fully elucidated. In the present study, miR-21 inhibitor and empty vector were transfected into OSCC cells, and non-transfected cells were used as a blank control. The results indicated that when compared with the control and scramble groups, miR-21 inhibitor suppressed the expression of miR-21. Conversely, phosphatase and tensin homolog deletion on chromosome 10 (PTEN) was markedly upregulated, and a dual luciferase reporter assay revealed PTEN to be a target gene of miR-21. Furthermore, miR-21 inhibitor decreased the proliferation and invasion and enhanced the apoptosis of OSCC cells. There was no significant difference in cell proliferation, invasion and apoptosis between the control and scramble groups. The present data suggested that there may be a regulatory loop between miR-21 and PTEN, and that miR-21 inhibition affected the proliferative, invasive and apoptotic abilities of OSCC cells. These findings indicate that miR-21 may be a possible novel target in the treatment of OSCC.
\end{abstract}

\section{Introduction}

In recent years, increasing data have indicated the importance of microRNAs (miRNAs) in the progression of cancers including oral squamous cell carcinoma (OSCC) (1-4). miRNAs are small non-coding RNAs of 22 nucleotides in length, existing as single stranded RNAs that act by binding to partially complementary sequences in the 3'untranslated regions (UTRs) of target gene mRNAs to regulate protein

Correspondence to: Dr Haiqing Ma, Department of Stomatology, Jining No. 1 People's Hospital, 6 Health Road, Jining, Shandong 272000, P.R. China

E-mail: 36761562@qq.com

*Contributed equally

Key words: miR-21, apoptosis, oral squamous cell carcinoma, phosphatase and tensin homolog deletion on chromosome 10 expression. miR-21, which is considered to be a proto-oncogene, is frequently reported as overexpressed in various cancer types and has been implicated in tumorigenesis $(5,6)$. In addition, miR-21 has been reported to serve an important role in several signaling pathways, including Wnt/ $\beta$-catenin and phosphatase and tensin homolog deletion on chromosome 10 (PTEN)/phosphatidylinositol 3-kinase (PI3K)/Akt $(7,8)$. Furthermore, miR-21 may negatively regulate multiple target genes, including programmed cell death protein 4 (PDCD-4), tissue inhibitor of metalloproteinases-3, integrin subunit $\beta 4$ and PTEN (9-12).

PTEN is a tumor suppressor gene that has frequently been reported to be involved in the regulation of cell proliferation, invasion, migration and apoptosis in many types of cancer, and the expression of PTEN is downregulated in a wide range of malignancies, including breast cancer, glioblastoma, colorectal carcinoma, pancreatic cancer and OSCC (13-17). Previous studies have demonstrated that the PI3K/Akt signaling pathway is involved in multiple biological processes and that PTEN functions as a tumor suppressor by negatively regulating the PI3K/Akt signaling pathway, which reduces cell growth and increases cell apoptosis $(18,19)$. miRNAs, including miR-136, miR-181a and miR-221/222, have been reported to regulate the expression of PTEN (20-22). However, the role of miR-21 with regard to the expression of PTEN in OSCC is not well established.

Since the therapeutic potential of miR-21 inhibitor, to the best of our knowledge, has not been investigated in OSCC, in the present study the biological effects and molecular mechanism of miR-21 involved in the apoptosis of OSCC cells were evaluated. The results obtained support that miR-21 inhibitor, via its influence on PTEN expression, may be a novel agent for the treatment of OSCC.

\section{Materials and methods}

Tissue acquisition. Surgically resected OSCC specimens were obtained from 35 patients (aged 45 to 70, including 19 males and 16 females) with OSCC between June 2015 and June 2017 at Jining No. 1 People's Hospital (Jining, China) with written informed consent. According to the Union for International Cancer Control (UICC) standards in 2002, all the cases were classified as phases I-IV. In addition, individual oral tissue samples from 10 normal subjects (aged 35-65, including 6 males and 4 females) were collected as controls 
with written informed consent. The experimental procedures were approved by the Research Ethics Committee of Jining No. 1 People's Hospital.

Hematoxylin and eosin $(H \& E)$ staining. $\mathrm{H} \& \mathrm{E}$ staining is a popular staining method in histology and it is one of the most widely used staining in medical diagnosis. The prepared 4- $\mu \mathrm{m}$ paraffin sections were incubated for $2 \mathrm{~h}$ in a $60^{\circ} \mathrm{C}$ incubator. The sections were deparaffinized by turpentine oil (TO) and sequentially soaked in 100, 95, 85 and 70\% alcohol solutions for $2 \mathrm{~min}$, and then hydrated using distilled water for $5 \mathrm{~min}$. The sections were stained with hematoxylin for 5-15 min. Then, the excess dyeing solution on the slide was washed with water. Next, the sections were stained with eosin for 1-5 min. Subsequently, the sections were dehydrated using 70, 85, 95 and $100 \%$ alcohol, respectively, and were transparent through TO. Finally, the excess TO transparent agent around the section was wiped off, and a suitable amount of neutral resin was added, and the slide was covered.

Immunohistochemical staining. Immunohistochemistry was used to detect PTEN expression in the OSCC specimens. The samples were fixed, embedded and cut into 4- $\mu \mathrm{m}$ thick sections. The sections were deparaffinized using xylene and rehydrated by increased grades of ethanol. A total of $0.5 \mu \mathrm{g}$ primary rabbit polyclonal antibodies against PTEN (1:200; cat. no. 9188S; Cell Signaling Technology, Inc., Danvers, MA, USA) were added following antigen retrieval and incubated at $4^{\circ} \mathrm{C}$ overnight. Subsequently, immunohistochemical staining was performed using VECTASTAIN ${ }^{\circledR}$ ABC immunohistochemistry kit (Cowin Biosciences, Co., Ltd., Beijing, China) according to the manufacturer's instructions.

In situ hybridization (ISH). ISH analysis was performed on deparaffinized 4- $\mu \mathrm{m}$ OSCC tissue sections using an ISH tissue implementation kit. OSCC sections were incubated at $60^{\circ} \mathrm{C}$ for $1 \mathrm{~h}$ and deparaffinized. Subsequently, the sections were rehydrated in decreasing concentrations of ethanol and washed in deionized water. The slides were incubated in $0.2 \mathrm{~mol} / 1 \mathrm{HCl}$ for $5 \mathrm{~min}$ at room temperature and washed 3 times in phosphate-buffered saline (PBS) for $5 \mathrm{~min}$. Proteins were digested for $15 \mathrm{~min}$ at $37^{\circ} \mathrm{C}$ following the addition of pepsin solution. Dehydration of sections was performed through incubation with 70, 95 and $100 \%$ ethanol for $1 \mathrm{~min}$ at each concentration. miR-21 probes labeled with digoxin were added to the hybrids. Sections were denatured at $90^{\circ} \mathrm{C}$ for $4 \mathrm{~min}$, followed by incubation in ISH slide denaturation and hybridization solution at $37^{\circ} \mathrm{C}$ overnight. Post-incubation, coverslips and glue were removed and the slides were washed. Finally, the slides were observed under an inverted microscope.

Cell culture and transfection. The OSCC cell lines SCC15 and SCC25 were obtained from the American Type Culture Collection (ATTC; Manassas, VA, USA) and cultured in Dulbecco's modified Eagle's medium (DMEM; Gibco; Thermo Fisher Scientific, Inc., Waltham, MA, USA) supplemented with $10 \%$ fetal bovine serum (FBS), $100 \mu \mathrm{g} / \mathrm{ml}$ penicillin, and $100 \mu \mathrm{g} / \mathrm{ml}$ streptomycin at $37^{\circ} \mathrm{C}$ in a humidified $5 \% \mathrm{CO}_{2}$ environment. miR-21 inhibitor and empty vector were chemically synthesized by Suzhou GenePharma Co., Ltd.
(Suzhou, China) and transfected into SCC15 and SCC25 cells using Lipofectamine ${ }^{\mathrm{TM}}$ RNAiMAX (Invitrogen; Thermo Fisher Scientific, Inc.) according to the recommended protocol. Cells in which Lipofectamine ${ }^{\mathrm{TM}}$ RNAiMAX was only added were used as a control group. Both SCC15 and SCC25 Cells in each of the 3 groups (control, scramble and miR-21 inhibitor) were collected to be used for subsequent analysis at different time-points following transfection.

Dual luciferase reporter assay. 293T cells were obtained from ATTC and cultured in 24-well plates and co-transfected with $0.5 \mu \mathrm{g}$ pMIR vectors containing PTEN 3'UTR or mutant (mut) PTEN 3'UTR and $20 \mu \mathrm{M}$ miR-21 mimics or $20 \mu \mathrm{M}$ miR-21 inhibitor using Lipofectamine ${ }^{\circledR}$ RNAiMAX. Cells were lysed with Passive Lysis Buffer and collected at $48 \mathrm{~h}$ post-transfection. Luciferase activity was detected with a dual luciferase reporter assay kit according to the manufacturer's protocol. Renilla luciferase activity was used for normalization.

Real-time reverse transcription-quantitative polymerase chain reaction ( $R T-q P C R)$ of miRNAs and mRNAs. Total miRNAs and mRNAs were extracted from SCC15 and SCC25 cells using an miRNeasy Mini kit (Qiagen $\mathrm{GmbH}$, Hilden, Germany) and Total RNA Purification kit (Qiagen $\mathrm{GmbH}$ ) according to the manufacturer's instructions. Real-time RT-qPCR analysis was performed to validate miRNA and mRNA expression using a One-Step RT-PCR Kit. U6 and glyceraldehyde 3-phosphate dehydrogenase (GAPDH; Invitrogen; Thermo Fisher Scientific, Inc.) were used as endogenous controls. Each test was repeated in triplicate.

Western blot analysis. After transfection for 48 h, SCC15 and SCC25 cells were washed 3 times with cold PBS and lysed in a buffer composed of radio-immunoprecipitation assay buffer (Cowin Biosciences Co., Ltd.) and phenylmethanesulfonyl fluoride (Cowin Biosciences Co., Ltd.) (100:1). Total proteins were heated at $95^{\circ} \mathrm{C}$ for $10 \mathrm{~min}$ following measurement of protein concentration with an Enhanced BCA Protein Assay kit (Cowin Biosciences Co., Ltd.). Equal quantities $(25 \mu \mathrm{g})$ of heated proteins from each sample were separated by $12 \%$ SDS-PAGE and transferred to polyvinylidene difluoride membranes. The blots were blocked in Tris-buffered saline with Tween-20 (TBST) containing 5\% non-fat milk at room temperature for $1 \mathrm{~h}$, then incubated with specific primary antibodies against against PTEN (1:1,000; cat. no. 9188S), phosphorylated (p)Akt (1:2,000; cat. no. 4060T), Akt (1:1,000; cat. no. 4691T) and GAPDH (1:1,000; cat. no. 5174S) (Cell Signaling Technology, Inc.) overnight at $4^{\circ} \mathrm{C}$. Subsequently, the blots were rinsed 3 times with TBST and incubated with the secondary antibody for $1 \mathrm{~h}$ at room temperature. Protein bands were imaged using the AlphaView SA Western blot detection system (Carl Zeiss AG, Oberkochen, Germany) and quantified following normalization to the density of GAPDH using Image J software (National Institutes of Health, Bethesda, MD, USA). Each test was repeated in triplicate.

Cell proliferation assay. Cell proliferation was assessed using Cell Counting Kit-8 (CCK-8; Cowin Biosciences Co., Ltd.) according to the manufacturer's instructions. Following treatment with miR-21 inhibitor, SCC15 and SCC25 cells were 
A

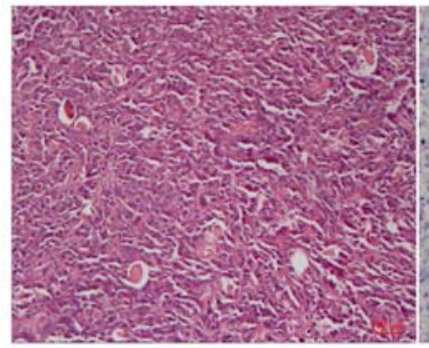

D

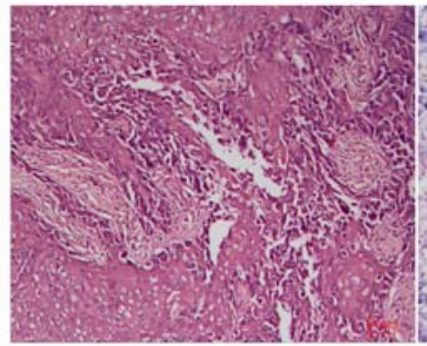

G

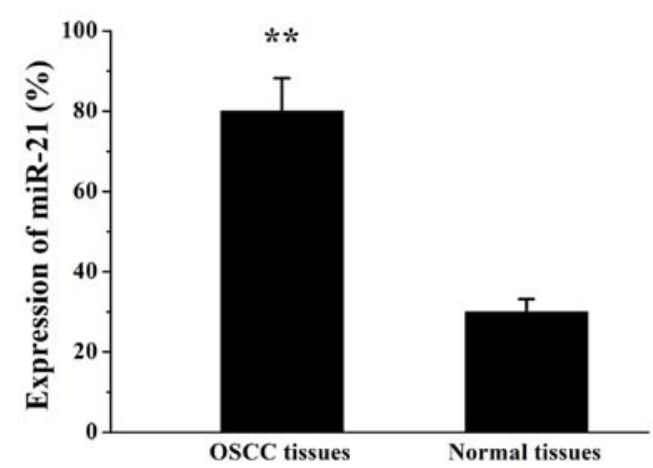

H
B

C

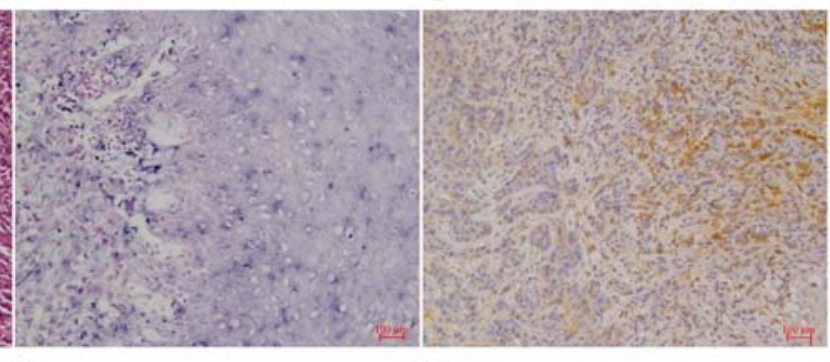

F

E
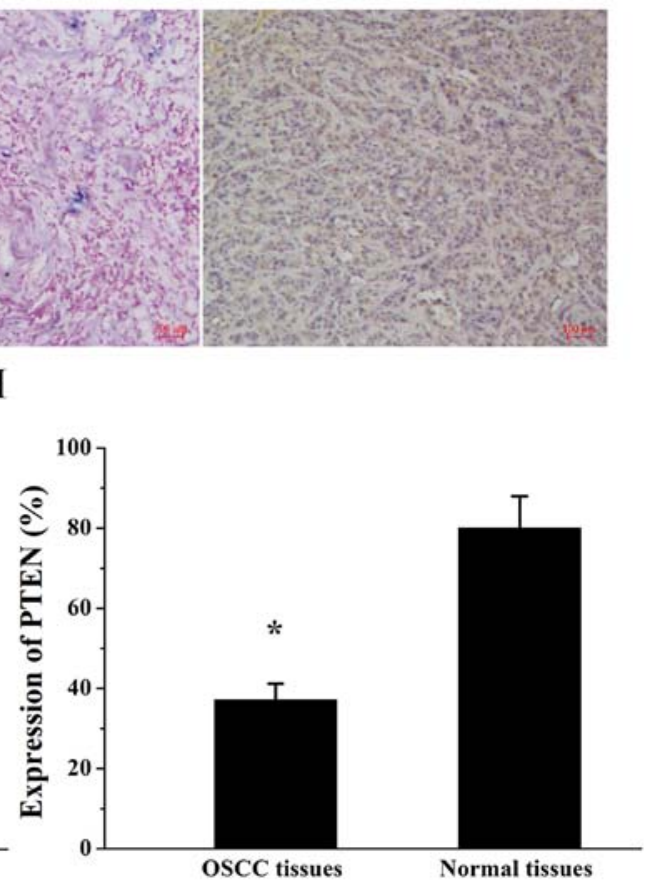

Figure 1. Expression of miR-21 and PTEN in OSCC samples. Hematoxylin and eosin staining results in (A) normal tissues and (D) OSCC tissues. miR-21 expression in (B) normal tissues and (E) OSCC tissues detected by in situ hybridization. Immunohistochemical staining of the expression of PTEN in (C) normal tissues and (F) OSCC tissues. (G and H) miR-21 and PTEN expression in OSCC and normal tissues. ${ }^{*} \mathrm{P}<0.05$ and ${ }^{* *} \mathrm{P}<0.01$. miR-21, microRNA-21; PTEN, phosphatase and tensin homolog deletion on chromosome 10; OSCC, oral squamous cell carcinoma.

seeded into 96-well plates at a density of 4,000 cells/well and subsequently allowed to attach overnight. A $10 \mu \mathrm{l}$ quantity of CCK-8 was added to each well at $0,24,48$ and $72 \mathrm{~h}$ after transfection, and the cells were incubated for $1 \mathrm{~h}$. Optical density (OD) was determined by spectrophotometric analysis at a wavelength of $450 \mathrm{~nm}$. Each experiment was repeated in triplicate.

Transwell cell migration assay. After transfection, $\mathrm{SCC} 15$ and SCC25 cells were transferred to $8-\mu \mathrm{m}$ pore inserts and placed in companion wells containing DMEM and 10\% FBS (Gibco; Thermo Fisher Scientific, Inc.). The inserts were removed after a 12-h incubation, and the non-migrated cells on the upper surface were harvested. Cells on the lower surface were fixed with 5\% glutaraldehyde (Beyotime Institute of Biotechnology, Shanghai, China) and stained with Giemsa (Beyotime Institute of Biotechnology) and counted under a fluorescent microscope. Each test was repeated in triplicate.

Cell apoptosis assay. SCC15 and SCC25 cells were washed with PBS and resuspended in buffer at a concentration of $10^{6}$ cells $/ \mathrm{ml}$. Cells were mixed with $5 \mu 1$ fluorescein isothiocyanate-conjugated Annexin $\mathrm{V}$ reagent and $5 \mu \mathrm{l}$ propidium iodide (PI) (Invitrogen; Thermo Fisher Scientific, Inc.). At 15 min after incubation in the dark at room temperature, the samples were analyzed by flow cytometry (Beckman Coulter GmbH, Krefeld, Germany). Each test was repeated in triplicate.

Statistical analysis. All experiments were repeated at least 3 times and the data were presented as the mean \pm standard deviation (SD). The results were analyzed by Student's t-test for the comparison of two and ANOVA (followed by Tukey's post hoc test) for the comparison of multiple samples using SPSS 19.0 software (IBM Corp., Armonk, NY, USA). P<0.05 was considered to indicate a statistically significant result.

\section{Results}

Expression of miR-21 and PTEN in OSCC tissues. The expression of miR-21 and PTEN was examined by ISH and immunohistochemical staining, respectively, in human OSCC tissues and normal tissues (Fig. 1). As depicted in Table I, miR-21 expression was observed in $80.0 \%$ (28/35) of OSCC 
Table I. miR-21 and PTEN expression in OSCC and normal tissues.

\begin{tabular}{|c|c|c|c|c|c|c|c|}
\hline \multirow[b]{2}{*}{ Group } & \multirow[b]{2}{*}{ No. of tumor specimens, $n$} & \multicolumn{3}{|c|}{ miR-21 expression } & \multicolumn{3}{|c|}{ PTEN expression } \\
\hline & & $\mathrm{n}$ & $\chi^{2}$ & P-value & $\mathrm{n}$ & $\chi^{2}$ & $\mathrm{P}$-value \\
\hline OSCC tissues & 35 & 28 & 9.073 & 0.003 & 13 & 5.740 & 0.017 \\
\hline Normal tissues & 10 & 3 & & & 8 & & \\
\hline
\end{tabular}

miR-21, microRNA-21; PTEN, phosphatase and tensin homolog deletion on chromosome 10; OSCC, in oral squamous cell carcinoma.

Table II. Associations of miR-21 and PTEN expression with clinicopathological profiles.

\begin{tabular}{|c|c|c|c|c|c|c|c|}
\hline \multirow[b]{2}{*}{ Clinicopathological profile } & \multirow[b]{2}{*}{ No. of tumor specimens, $\mathrm{n}$} & \multicolumn{3}{|c|}{ miR-21 expression } & \multicolumn{3}{|c|}{ PTEN expression } \\
\hline & & $\mathrm{n}$ & $\chi^{2}$ & P-value & $\mathrm{n}$ & $\chi^{2}$ & P-value \\
\hline \multicolumn{8}{|l|}{ Sex } \\
\hline Male & 19 & 16 & 0.461 & 0.497 & 8 & 0.438 & 0.508 \\
\hline Female & 16 & 12 & & & 5 & & \\
\hline \multicolumn{8}{|l|}{ Age, years } \\
\hline$>60$ & 20 & 17 & 0.729 & 0.393 & 7 & 0.092 & 0.762 \\
\hline$\leq 60$ & 15 & 11 & & & 6 & & \\
\hline \multicolumn{8}{|l|}{ Stage } \\
\hline $\mathrm{I}+\mathrm{II}$ & 17 & 11 & 4.833 & 0.028 & 10 & 6.655 & 0.010 \\
\hline III+IV & 18 & 17 & & & 3 & & \\
\hline \multicolumn{8}{|l|}{ Differentiation } \\
\hline Well & 9 & 7 & 0.043 & 0.979 & 3 & 0.092 & 0.955 \\
\hline Moderate & 16 & 13 & & & 6 & & \\
\hline Poor & 10 & 8 & & & 4 & & \\
\hline \multicolumn{8}{|l|}{ Lymph node metastasis } \\
\hline Negative & 23 & 18 & 0.127 & 0.722 & 9 & 0.114 & 0.736 \\
\hline Positive & 12 & 10 & & & 4 & & \\
\hline Total & 35 & 28 & & & 13 & & \\
\hline
\end{tabular}

miR-21, microRNA-21; PTEN, phosphatase and tensin homolog deletion on chromosome 10.

tissues and in $30.0 \%(3 / 10)$ of normal tissues $(\mathrm{P}=0.003)$. By contrast, PTEN expression exhibited an opposite trend in OSCC tissues $(37.1 \%, 13 / 35)$ and normal tissues $(80.0 \%$, $8 / 10 ; \mathrm{P}=0.017)$ compared with the expression of miR-21. Furthermore, the association between miR-21 and PTEN expression and the clinicopathological profiles of patients was evaluated (Table II). The data indicated that the expression of miR-21 and PTEN was associated with tumor stage. miR-21 expression was lower in early stages (I+II) than in advanced stages (III+IV; $\mathrm{P}=0.028)$ and the expression of PTEN was significantly higher in early stages (I+II) than in advanced stages (III+IV; $\mathrm{P}=0.010)$. miR-21 and PTEN expression levels were not significantly associated with patient sex or age, tumor differentiation or lymph node metastases $(\mathrm{P}>0.05)$.

miR-21 and PTEN expression in SCC15 and SCC25 cells. To knockdown endogenous miR-21, miR-21 inhibitor was synthesized and transfected into SCC15 and SCC25 cells. The expression of miR-21 and PTEN was then analyzed by RT-qPCR. The data indicated that miR-21 inhibitor efficiently silenced the expression of miR-21 compared with that in the control groups (Fig. 2A; $\mathrm{P}<0.01$ ). By contrast, the gene expression of PTEN was notably upregulated in the miR-21 inhibitor group (Fig. 2B; $\mathrm{P}<0.01$ ). No significant differences between the scramble and control groups were noted $(\mathrm{P}>0.05)$. The results indicated that miR-21 may be negatively associated with PTEN expression in SCC15 and SCC25 cells.

miR-21 inhibitor transfection altered the expression of PTEN, Akt and pAkt. To investigate the molecular mechanism of miR-21 in the apoptosis of SCC15 and SCC25 cells, western blot analysis was performed following miR-21 silencing to assess the expression of PTEN, Akt and pAkt. The expression of PTEN was significantly increased $(\mathrm{P}<0.01)$ and the 

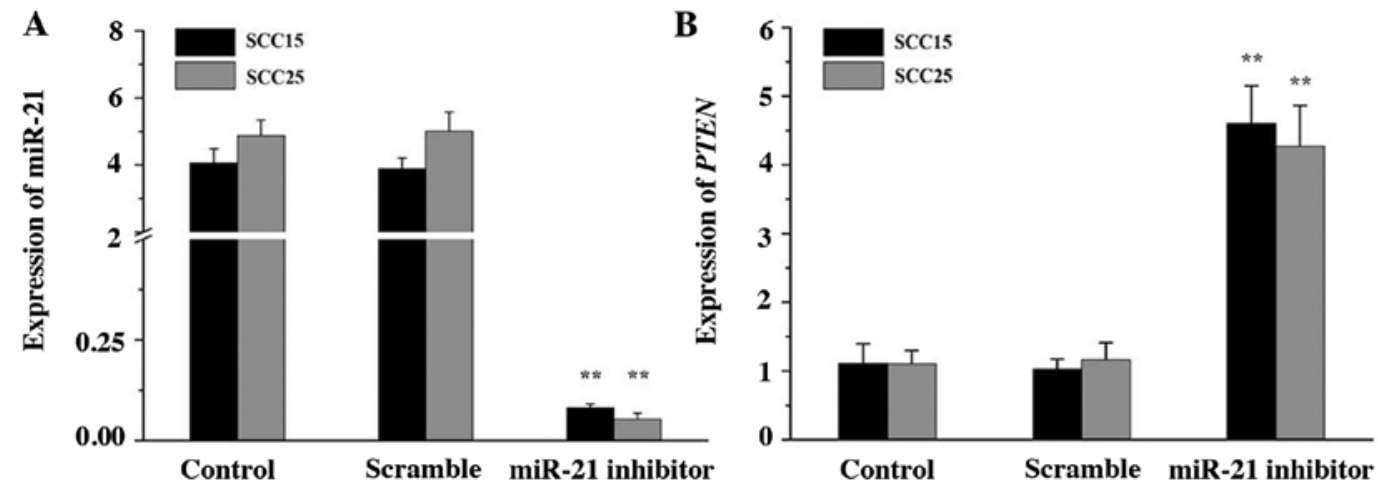

Figure 2. Reverse transcription-quantitative polymerase chain reaction analysis of the expression of (A) miR-21 and (B) PTEN in SCC15 and SCC25 cells transfected with miR-21 inhibitor. The results demonstrated significant downregulation of miR-21 and marked upregulation of PTEN in the miR-21 inhibitor groups compared with those in the control groups in both SCC15 and SCC25 cells. ${ }^{* *} \mathrm{P}<0.01$. miR-21, microRNA-21; PTEN, phosphatase and tensin homolog deletion on chromosome 10.

A
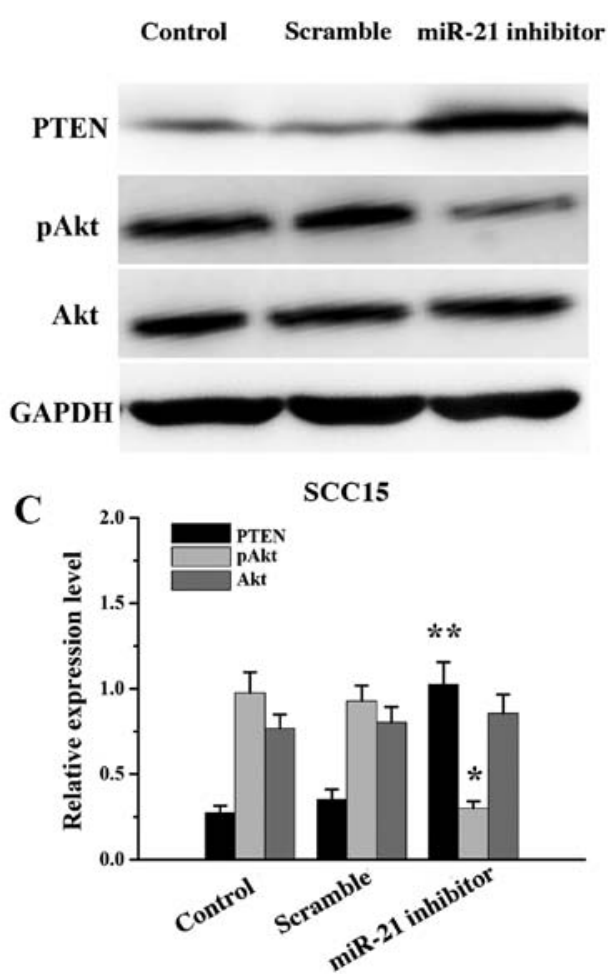

B

SCC25

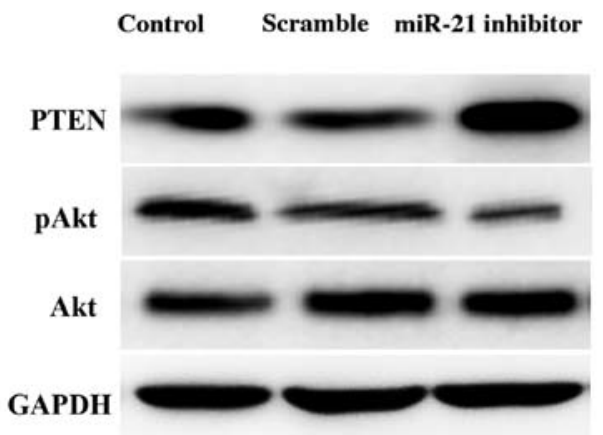

D

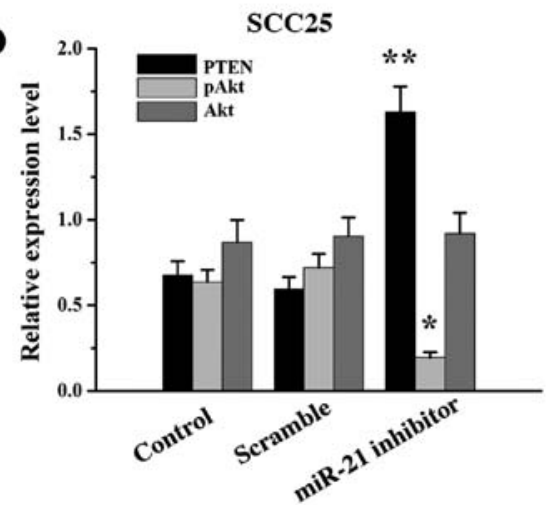

Figure 3. (A-D) Influence of miR-21 silencing on PTEN, pAkt and Akt signaling. Expression of PTEN, pAkt and Akt in (A and C) SCC15 and (B and D) SCC25 cells determined by western blot analysis following treatment with miR-21 inhibitor or empty vector. PTEN was observed to be overexpressed in the miR-21-inhibitor groups compared with that in the control groups in both SCC15 and SCC25 cells. By contrast, when compared with the expression of PTEN, pAkt expression exhibited an opposite trend. ${ }^{*} \mathrm{P}<0.05$ and ${ }^{* *} \mathrm{P}<0.01$. miR-21, microRNA-21; PTEN, phosphatase and tensin homolog deletion on chromosome 10; p, phosphorylated.

expression of pAkt decreased $(\mathrm{P}<0.05)$ in the miR-21 inhibitor groups compared with that in the control groups. There were no significant differences in Akt expression between the miR-21 inhibitor and control groups ( $P>0.05$; Fig. 3$)$. The results indicated that the level of PTEN expression in SCC15 and SCC25 cells was negatively regulated by miR-21.

miR-21 silencing inhibits SCC15 and SCC25 cell proliferation and invasion. The effect of miR-21 inhibitor on the proliferation and invasion of SCC15 and SCC25 cells was determined by CCK-8 and Transwell assays, respectively. As depicted in Fig. 4, both the proliferative and invasive abilities of SCC15 and SCC25 cells in the miR-21 inhibitor groups were significantly suppressed compared with those in the control groups $(\mathrm{P}<0.05)$. There were no significant differences between the control and scramble groups $(\mathrm{P}>0.05)$.

miR-21 inhibitor induces SCC15 and SCC25 cell apoptosis. To detect the effect of miR-21 inhibitor on SCC15 and SCC25 cell apoptosis, Annexin V/PI analysis was performed. The data indicated that the percentage of apoptotic cells was markedly induced in the miR-21 inhibitor groups compared with that 
A
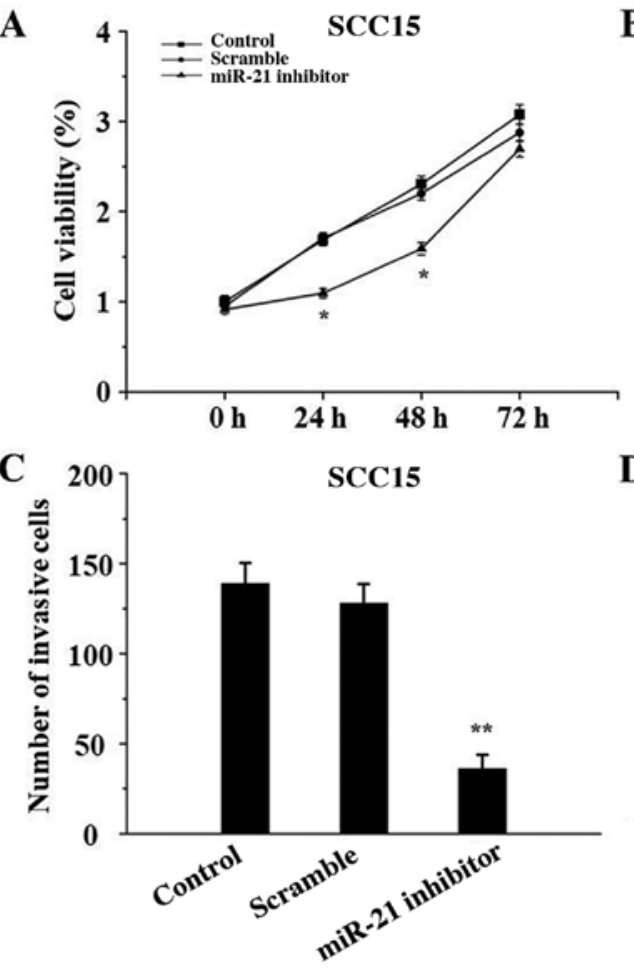

B
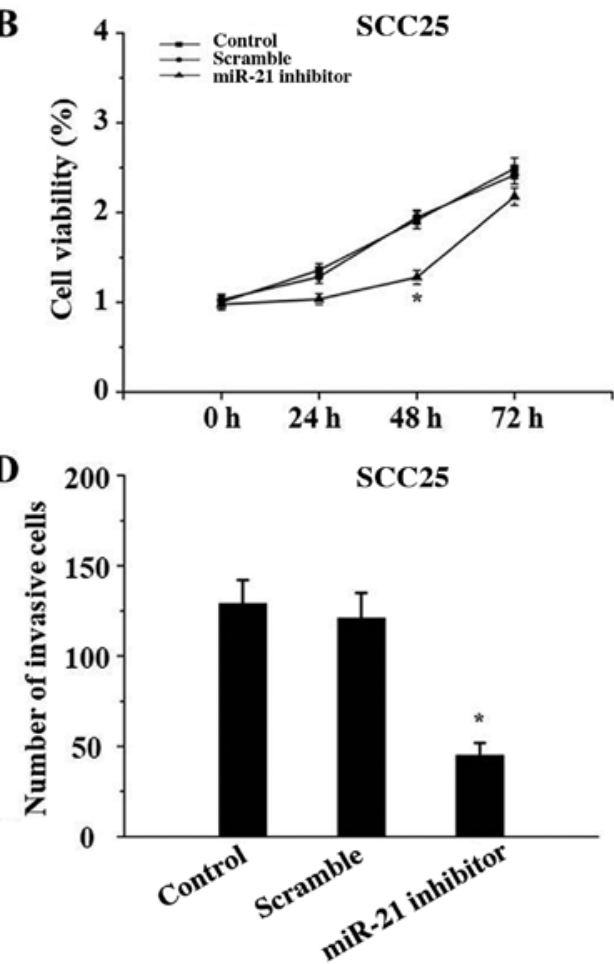

Figure 4. (A-D) miR-21 influences SCC15 and SCC25 cell proliferation and invasion. Cell Counting Kit-8 and Transwell assays indicated that in (A and C) SCC15 and (B and D) SCC25 cells, miR-21 silencing significantly inhibited cell proliferation and invasion compared with those in the control groups. ${ }^{*}<<0.05$ and ${ }^{* *} \mathrm{P}<0.01$. miR-21, microRNA-21.
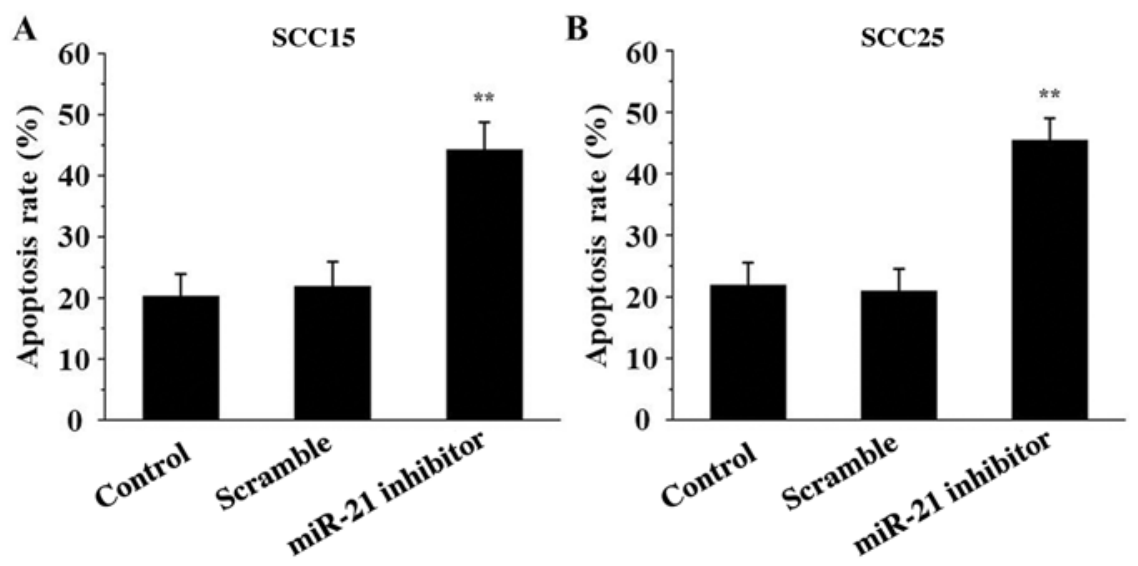

Figure 5. Percentages of apoptotic SCC15 and SCC25 cells following treatment with miR-21 inhibitor. The apoptotic rates of (A) SCC15 and (B) SCC25 cells were analyzed by flow cytometry at $48 \mathrm{~h}$ post-treatment with miR-21 inhibitor or empty vector. The data indicated that the percentage of apoptotic cells was notably increased in the miR-21-inhibitor groups compared with that in the control and scramble groups. ${ }^{* *} \mathrm{P}<0.01$. miR-21, microRNA-21.

in the control groups $(\mathrm{P}<0.01$; Fig. 5), indicating that miR-21 inhibitor transfection induced cell apoptosis.

miR-21 acts directly on PTEN mRNA 3'UTR. To validate whether PTEN is a direct target of miR-21, PTEN 3'UTR and mutPTEN 3'UTR luciferase constructs were transfected into 293T cells with negative control (NC) mimics, miR-21 mimics, inhibitor NC or miR-21 inhibitor. Luciferase activity was assessed using a dual-luciferase reporter assay system. As illustrated in Fig. 6, compared with the cells in the other groups, the luciferase activity of 293T cells transfected with miR-21 mimics and PTEN 3'UTR was significantly reduced $(\mathrm{P}<0.05)$.

\section{Discussion}

OSCC is the most common cancer of the head and neck and presents a poor prognosis, with a 5 -year survival rate of $<60 \%(23,24)$. As one of the current treatment strategies for OSCC, gene therapy serves an important role alongside other treatment modalities including surgery, chemotherapy and radiotherapy. However, poor target activity is a pivotal reason that restricts the clinical applications of gene therapy in treating cancers. Therefore, there is a critical need to identify sensitive gene targets and to understand the molecular mechanism involved in the aggressive growth characteristics of OSCC. 
A

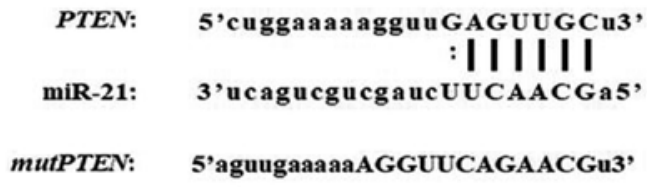

B

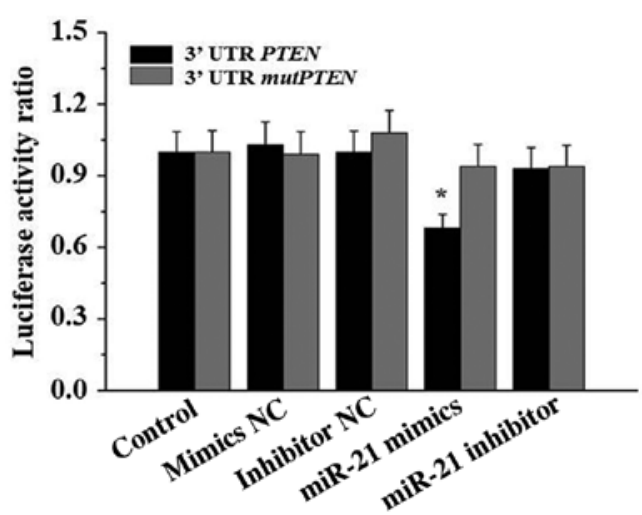

Figure 6. PTEN was identified as a target gene of miR-21. (A) Sequences of miR-21, PTEN 3'UTR and mutPTEN 3'UTR, including the sites involved in the binding between PTEN 3'UTR and miR-21. (B) Luciferase activity ratio was detected $48 \mathrm{~h}$ after 293T cells were transfected with mimics NC, inhibitor NC, miR-21 mimics or miR-21 inhibitor. "P $<0.05$. miR-21, microRNA-21; PTEN, phosphatase and tensin homolog deletion on chromosome 10; UTR, untranslated region; mut, mutant; $\mathrm{NC}$, negative control.

miRNAs are considered to be important regulators of cell proliferation, differentiation, the cell cycle and cell death. They are also considered to be novel molecular targets in the diagnosis and treatment of human carcinomas. As a member of the oncomiRs, miR-21 has been confirmed to be overexpressed in various types of tumors and to be capable of negatively regulating multiple target genes. Li et al (25) demonstrated that miR-21 was overexpressed in tongue SCC (TSCC) tissues compared with that in the adjacent normal tissues, and that it may regulate TSCC development by inhibiting TSCC cell apoptosis in part via tropomyosin $\alpha-1$ chain silencing. Koenig et al (26) compared the expression of miR-21 targets in 377 patients with liver cancer and revealed that the levels of 402 miR-21 targets were altered in hepatocellular carcinoma. Their analysis identified novel miR-21 targets (CAMSAP1, DDX1, MARCKSL1 and RMND5A) that appeared likely to serve a causal role in hepatocarcinogenesis. Yan et al (27) reported that miR-21 may promote salivary adenoid cystic carcinoma (SACC) progression through PDCD-4, PTEN and B-cell lymphoma 2, and suggested that miR-21 may be a novel target for SACC therapy.

The present study on OSCC determined that the expression of miR-21 had a negative association with the expression of PTEN protein. In OSCC cells, real-time RT-qPCR results revealed that the expression of miR-21 was significantly reduced in SCC15 and SCC25 cells transfected with miR-21 inhibitor compared with that in the control groups. By contrast, upregulation of PTEN gene expression was observed following the treatment with miR-21 inhibitor. The data indicated that miR-21 may negatively regulate PTEN gene expression in both OSCC tissues and cells. Furthermore, bioinformatics and luciferase assays indicated that miR-21 modulates PTEN expression by directly targeting a binding site within the mRNA 3'UTR. Collectively these findings revealed that PTEN is directly regulated by miR-21.

As the first tumor suppressor gene identified with phosphatase activity, PTEN has been confirmed as a target gene in colorectal, gastric, cervical and non-small cell lung cancer. Wu et al (28) reported that miR-21 could modulate malignant phenotypes including proliferation, invasion, cell cycle progression and anti-apoptosis in colorectal cancer cells by downregulating PTEN protein expression. A previous study indicated that the PI3K/Akt signaling pathway was activated in multiple types of cancers, and notably that the mechanisms activating PI3K/Akt signaling included loss of function of PTEN (29). Wang et al (30) observed that miR-155 suppressed PTEN expression, enhanced PI3K/Akt/mTOR signaling and inhibit human osteosarcoma MG-63 cell apoptosis and autophagy was induced by adrenomedullin. In the present study, upregulation of PTEN and downregulation of pAkt proteins was observed following treatment with miR-21 inhibitor. Additionally, the results revealed that when the expression of miR-21 was suppressed, the proliferative and invasive abilities of SCC15 and SCC25 cells were inhibited, while cell apoptosis was promoted. These data indicated that cell proliferation, invasion and apoptosis may be associated with the expression of PTEN and the PI3K/Akt signaling pathway in OSCC cell lines.

In conclusion, the present study revealed that miR-21 inhibitor transfection significantly inhibited the growth of SCC15 and $\mathrm{SCC} 25$ cells by reducing cell proliferation and promoting cell apoptosis. It was also revealed by luciferase assay that PTEN was a direct target of miR-21. Furthermore, the results indicated that the modulation of miR-21 activity may regulate the expression of PTEN, and that the PI3K/Akt signaling pathway may be involved through this targeting of PTEN. Overall, these present results indicate that the miR-21/PTEN axis may be a potential novel therapeutic target in OSCC.

\section{Acknowledgements}

The authors thank Mr. Shenghui Yang for his technical support.

\section{Funding}

The present study was supported by the Shandong Medical and Health Technology Development Project (grant no. 2017WS343).

\section{Availability of data and materials}

The datasets used during the present study are available from the corresponding author upon reasonable request. 


\section{Authors' contributions}

YZ, JX and HM conceived and designed the study. JX, FJ, YL and GC performed the experiments. YL, GC and HM analyzed the data. YZ and FJ wrote the manuscript. All authors read and approved the manuscript and agree to be accountable for all aspects of the research in ensuring that the accuracy or integrity of any part of the work are appropriately investigated and resolved.

\section{Ethics approval and consent to participate}

The experimental procedures were approved by the Research Ethics Committee of Jining No. 1 People's Hospital and written informed consent was obtained from all participants.

\section{Patient consent for publication}

Not applicable.

\section{Competing interests}

The authors declare that they have no competing interests.

\section{References}

1. Shi Z, Li Y, Qian X, Hu Y, Liu J, Zhang S and Zhang J: MiR-340 inhibits triple-negative breast cancer progression by reversing EZH2 mediated miRNAs dysregulated expressions. J Cancer 8 3037-3048, 2017.

2. Hawa Z, Haque I, Ghosh A, Banerjee S, Harris L and Banerjee SK: The miRacle in pancreatic cancer by miRNAs: Tiny angels or devils in disease progression. Int J Mol Sci 17: E809, 2016.

3. Zhang M, Matyunina LV, Walker LD, Chen W, Xiao H, Benigno BB, Wu R and McDonald JF: Evidence for the importance of post-transcriptional regulatory changes in ovarian cancer progression and the contribution of miRNAs. Sci Rep 7: 8171, 2017

4. Pereira CM, Sehnem D, da Fonseca EO, Barboza HFG, de Carvalho ACP, DaSilva AFM, Moura-Neto V and DosSantos MF: miRNAs: Important targets for oral cancer pain research. Biomed Res Int 2017: 4043516, 2017.

5. Li L, Wang X, Li W, Yang L, Liu R, Zeng R, Wu Y and Shou T: miR-21 modulates prostaglandin signaling and promotes gastric tumorigenesis by targeting 15-PGDH. Biochem Biophys Res Commun 495: 928-934, 2018.

6. Yang Q, Xu E, Dai J, Wu J, Zhang S, Peng B and Jiang Y: miR-21 regulates $N$-methyl- $N$-nitro- $N$ '-nitrosoguanidine-induced gastric tumorigenesis by targeting FASLG and BTG2. Toxicol Lett 228: 147-156, 2014

7. Zhou B, Wang J, Zheng G and Qiu Z: Methylated urolithin A, the modified ellagitannin-derived metabolite, suppresses cell viability of DU145 human prostate cancer cells via targeting miR-21. Food Chem Toxicol 97: 375-384, 2016.

8. Wang P, Guan Q, Zhou D, Yu Z, Song Y and Qiu W: miR-21 inhibitors modulate biological functions of gastric cancer cells via PTEN/PI3K/mTOR pathway. DNA Cell Biol 37: 38-45, 2018.

9. Frankel LB, Christoffersen NR, Jacobsen A, Lindow M, Krogh A and Lund AH: Programmed cell death 4 (PDCD4) is an important functional target of the microRNA miR-21 in breast cancer cells. J Biol Chem 283: 1026-1033, 2008.

10. Zhou S, Zhang S, Wang Y, Yi S, Zhao L, Tang X, Yu B, Gu X and Ding F: MiR-21 and miR-222 inhibit apoptosis of adult dorsal root ganglion neurons by repressing TIMP3 following sciatic nerve injury. Neurosci Lett 586: 43-49, 2015.

11. Ferraro A, Kontos CK, Boni T, Bantounas I, Siakouli D, Kosmidou V, Vlassi M, Spyridakis Y, Tsipras I, Zografos G, et al: Epigenetic regulation of miR-21 in colorectal cancer: ITGB4 as a novel miR-21 target and a three-gene network (miR-21-ITGBeta4-PDCD4) as predictor of metastatic tumor potential. Epigenetics 9: 129-141, 2014.
12. Chanyshev MD, Ushakov DS and Gulyaeva LF: Expression of $m i R-21$ and its Acat1, Armcxl, and PTEN target genes in liver of female rats treated with DDT and benzo[a]pyrene. Mol Biol 51: 664-670, 2017 (In Russian).

13. Guo Y, Chang H, Li J, Xu XY, Shen L, Yu ZB and Liu WC: Thymosin alpha 1 suppresses proliferation and induces apoptosis in breast cancer cells through PTEN-mediated inhibition of PI3K/Akt/mTOR signaling pathway. Apoptosis 20: 1109-1121, 2015.

14. Li XT, Wang HZ, Wu ZW, Yang TQ, Zhao ZH, Chen GL, Xie XS, Li B, Wei YX, Huang YL, et al: miR-494-3p regulates cellular proliferation, invasion, migration, and apoptosis by PTEN/AKT signaling in human glioblastoma cells. Cell Mol Neurobiol 35: 679-687, 2015.

15. Li Y, Sun J, Cai Y, Jiang Y, Wang X, Huang X, Yin Y and Li H: MiR-200a acts as an oncogene in colorectal carcinoma by targeting PTEN. Exp Mol Pathol 101: 308-313, 2016.

16. Gao ZQ, Wang JF, Chen DH, Ma XS, Wu Y, Tang Z and Dang XW: Long non-coding RNA GAS5 suppresses pancreatic cancer metastasis through modulating miR-32-5p/PTEN axis. Cell Biosci 7: 66, 2017.

17. Sushma PS, Jamil K, Kumar PU, Satyanarayana U, Ramakrishna M and Triveni B: PTEN and p16 genes as epigenetic biomarkers in oral squamous cell carcinoma (OSCC): A study on south Indian population. Tumour Biol 37: 7625-7632, 2016.

18. Nan Y, Guo L, Song Y, Wang L, Yu K, Huang Q and Zhong Y: Combinatorial therapy with adenoviral-mediated PTEN and a PI3K inhibitor suppresses malignant glioma cell growth in vitro and in vivo by regulating the PI3K/AKT signaling pathway. J Cancer Res Clin Oncol 143: 1477-1487, 2017.

19. Zhu X, Li Z, Li T, Long F, Lv Y,Liu L, Liu X and Zhan Q: Osthole inhibits the PI3K/AKT signaling pathway via activation of PTEN and induces cell cycle arrest and apoptosis in esophageal squamous cell carcinoma. Biomed Pharmacother 102: 502-509, 2018.

20. Chen X, Huang Z and Chen R: Microrna-136 promotes proliferation and invasion ingastric cancer cells through Pten/Akt/P-Akt signaling pathway. Oncol Lett 15: 4683-4689, 2018.

21. Geletina NS, Kobelev VS, Babayants EV, Feng L, Pustylnyak VO and Gulyaeva LF: PTEN negative correlates with miR-181a in tumour tissues of non-obese endometrial cancer patients. Gene 655: 20-24, 2018.

22. Li B, Lu Y, Yu L, Han X, Wang H, Mao J, Shen J, Wang B, Tang J, Li C and Song B: miR-221/222 promote cancer stem-like cell properties and tumor growth of breast cancer via targeting PTEN and sustained Akt/NF- $\kappa \mathrm{B} / \mathrm{COX}-2$ activation. Chem Biol Interact 277: 33-42, 2017.

23. Taghavi $\mathrm{N}$ and Yazdi I: Prognostic factors of survival rate in oral squamous cell carcinoma: Clinical, histologic, genetic and molecular concepts. Arch Iran Med 18: 314-319, 2015.

24. Tamaki S, Kawakami M, Ishitani A, Kawashima W, Kasuda S, Yamanaka Y, Shimomura H, Imai Y, Nakagawa Y, Hatake K, et al: Soluble MICB serum levels correlate with disease stage and survival rate in patients with oral squamous cell carcinoma. Anticancer Res 30: 4097-4101, 2010.

25. Li J, Huang H, Sun L, Yang M, Pan C, Chen W, Wu D, Lin Z, Zeng C, Yao Y, et al: MiR-21 indicates poor prognosis in tongue squamous cell carcinomas as an apoptosis inhibitor. Clin Cancer Res 15: 3998-4008, 2009.

26. Koenig AB, Barajas JM, Guerrero MJ and Ghoshal K: A comprehensive analysis of argonaute-clip data identifies novel, conserved and species-specific targets of mir-21 in human liver and hepatocellular carcinoma. Int J Mol Sci 19: E851, 2018.

27. Yan F, Wang C, Li T, Cai W and Sun J: Role of miR-21 in the growth and metastasis of human salivary adenoid cystic carcinoma. Mol Med Rep 17: 4237-4244, 2018.

28. Wu Y, Song Y, Xiong Y, Wang X, Xu K, Han B, Bai Y, Li L, Zhang Y and Zhou L: MicroRNA-21 (Mir-21) promotes cell growth and invasion by repressing tumor suppressor PTEN in colorectal cancer. Cell Physiol Biochem 43: 945-958, 2017.

29. Jung S, Li C, Jeong D, Lee S, Ohk J, Park M, Han S, Duan J, Kim C, Yang Y, et al: Oncogenic function of p34 $4^{\text {SEI-1 }}$ via NEDD41 mediated PTEN ubiquitination/degradation and activation of the PI3K/AKT pathway. Int J Oncol 43: 1587-1595, 2013.

30. Wang L, Tang B, Han H, Mao D, Chen J, Zeng Y and Xiong M: miR-155 affects osteosarcoma MG-63 cell autophagy induced by adriamycin through regulating PTEN-PI3K/AKT/mTOR signaling pathway. Cancer Biother Radiopharm 33: 32-38, 2018. 